\title{
Single Photon Extraction for FACT's SiPMs allows for Novel IACT Event Representation
}

\author{
S. A. Mueller ${ }^{* a}$, J. Adam ${ }^{b}$, M. L. Ahnen ${ }^{a}$, D. Baack ${ }^{b}$, M. Balbo ${ }^{c}$, A. Biland ${ }^{a}$, \\ M. Blank ${ }^{d}$, T. Bretz ${ }^{a, 1}$, K. Bruegge ${ }^{b}$, J. Buss ${ }^{b}$, A. Dmytriiev ${ }^{c}$, D. Dorner ${ }^{d}$, \\ S. Einecke ${ }^{b}$, D. Elsaesser ${ }^{b}$, C. Hempfling ${ }^{d}$, T. Herbst ${ }^{d}$, D. Hildebrand ${ }^{a}$, \\ L. Kortmann $^{b}$, L. Linhoff ${ }^{b}$, M. Mahlke ${ }^{a, 1}$, K. Mannheim ${ }^{d}$, D. Neise ${ }^{a}$, A. Neronov ${ }^{c}$, \\ M. Noethe ${ }^{b}$, J. Oberkirch ${ }^{b}$, A. Paravac ${ }^{d}$, F. Pauss ${ }^{a}$, W. Rhode ${ }^{b}$, B. Schleicher ${ }^{d}$,

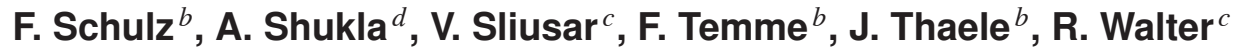 \\ Email: sebmuellephys.ethz.ch
}

${ }^{a}$ ETH Zurich, Institute for Particle Physics Otto-Stern-Weg 5, 8093 Zurich, Switzerland

${ }^{b}$ TU Dortmund, Experimental Physics 5

Otto-Hahn-Str. 4, 44221 Dortmund, Germany

${ }^{c}$ University of Geneva, ISDC Data Center for Astrophysics

Chemin d'Ecogia 16, 1290 Versoix, Switzerland

${ }^{d}$ Universität Würzburg, Institute for Theoretical Physics and Astrophysics

Emil-Fischer-Str. 31, 97074 Würzburg, Germany

${ }^{1}$ also at RWTH Aachen University

\begin{abstract}
Imaging Atmospheric Cherenkov Telescopes provide large gamma-ray collection areas $>10^{4} \mathrm{~m}^{2}$ and successfully probe the high energetic gamma-ray sky by observing extensive air-showers during the night. The First G-APD Cherenkov Telescope (FACT) explores silicon based photoelectric converters (called G-APDs or SiPMs) which provide more observation time with strong moonlight, a more stable photon gain over years of observations, and mechanically simpler imaging cameras. So far, the signal extraction methods used for FACT originate from sensors with no intrinsic quantized responses like photomultiplier tubes. This standard signal extraction is successfully used for the long time monitoring of the gamma-ray flux of bright blazars. However, we now challenge our classic signal extraction and explore single photon extraction methods to take advantage of the highly stable and quantized single photon responses of FACT's SiPM sensors. Instead of having one main pulse with one arrival time and one photon equivalent extracted for each pixel, we extract the arrival times of all individual photons in a pixel's time line which opens up a new dimension in time for representing extensive air-showers with an IACT.
\end{abstract}

35th International Cosmic Ray Conference - ICRC2017

10-20 July, 2017

Bexco, Busan, Korea

${ }^{*}$ Speaker. 


\section{Introduction}

FACT is the first atmospheric Cherenkov gamma-ray telescope to use silicon photo sensors (SiPMs) instead of classic photo multiplier tubes [1]. FACT uses a $9.5 \mathrm{~m}^{2}$ imaging reflector and an SiPM camera to record air-showers induced by cosmic particles. FACT reconstructs the direction and energy of these individual particles to do gamma-ray astronomy. FACT's camera has $4.5^{\circ}$ field of view and consists out of 1440 SiPM pixels. Each pixel has $0.1^{\circ}$ field of view, see Figure 1. Each
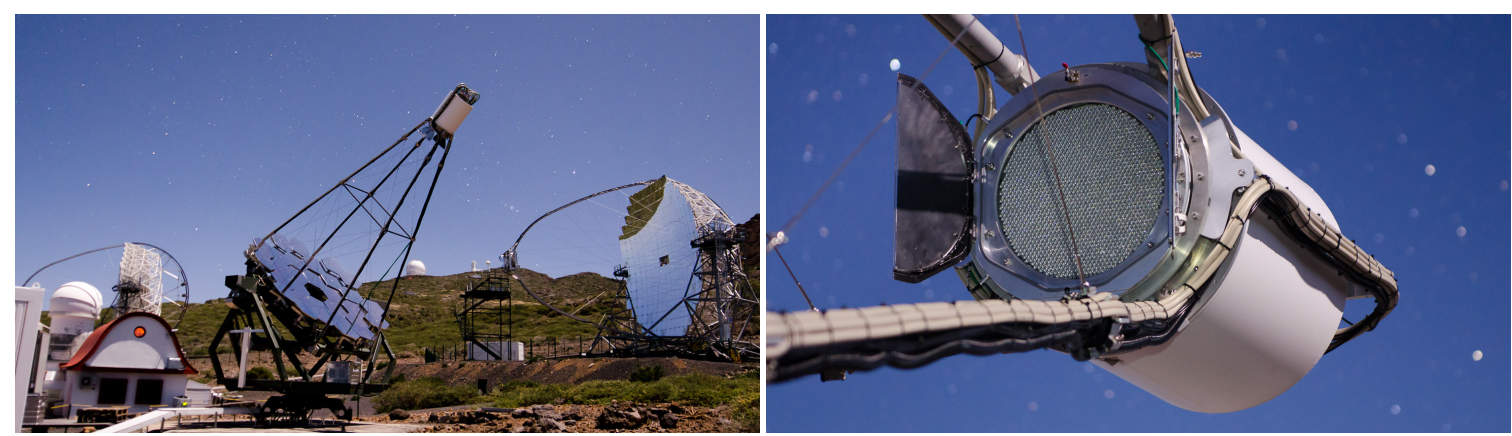

Figure 1: The FACT gamma-ray telescope on Canary Island La Palma, Spain. And the camera of FACT with its lid open. Photographs by Thomas Kraehenbuehl.

SiPM pixel consists out of 3600 Geiger mode avalanche photo diodes connected in parallel. The output of an SiPM pixel is sampled in a switched capacitor array and read out when a trigger criteria is fulfilled. The amplitude time line of a pixel contains the pulses of the discharges of the avalanche photo diodes when these are hit by a photon. So far, FACT identifies the main pulse on these time lines to extract the arrival time $t$ and the photon equivalent $C$ for each pixel, Figures 2 and 3 .

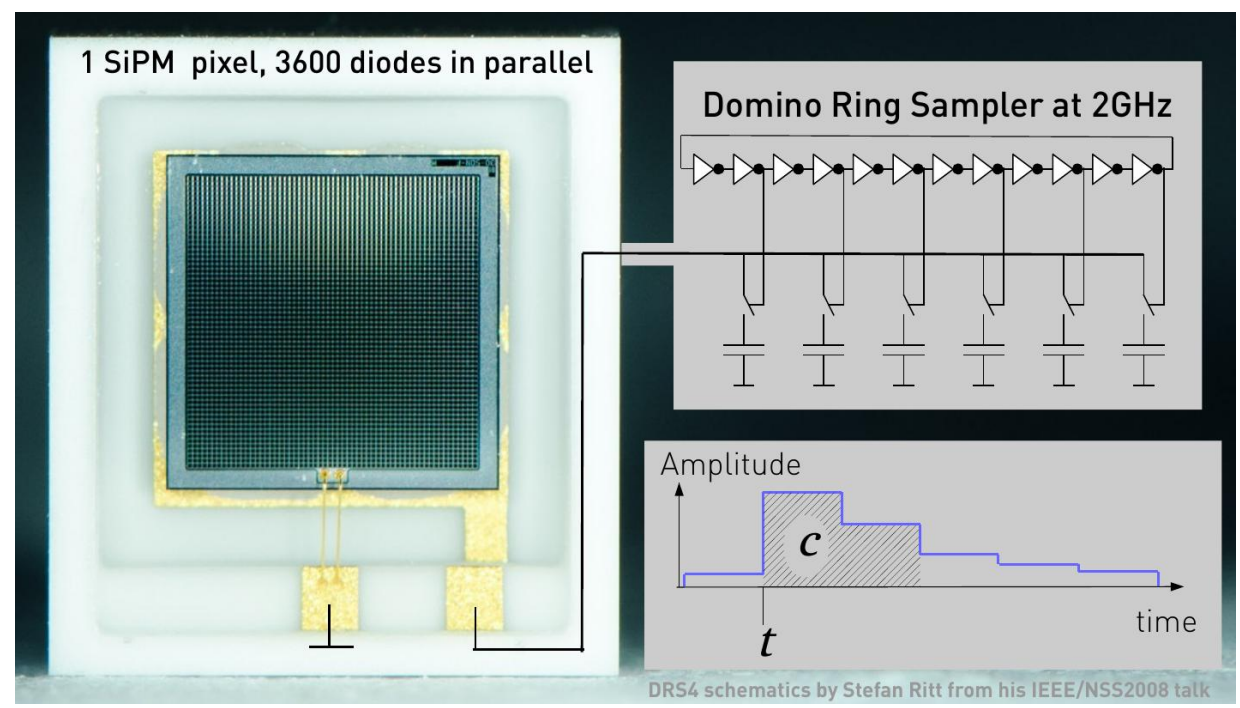

Figure 2: The electric flow chart of readout electronics in FACT. Incoming photons add up their pulses on an electric amplitude time line. The SiPM part designation is Hamamatsu MPPC S1036233-50C. An event in FACT is sampled in 300 capacitors over $150 \mathrm{~ns}$. 
To have a more detailed event representation that might improve air-shower reconstruction and gamma hadron separation, FACT now challenges this classic event representation and proposes to take advantage of the highly stable single photon gain [2] of its SiPMs. To do so, we implement a single photon extractor dedicated to SiPMs.
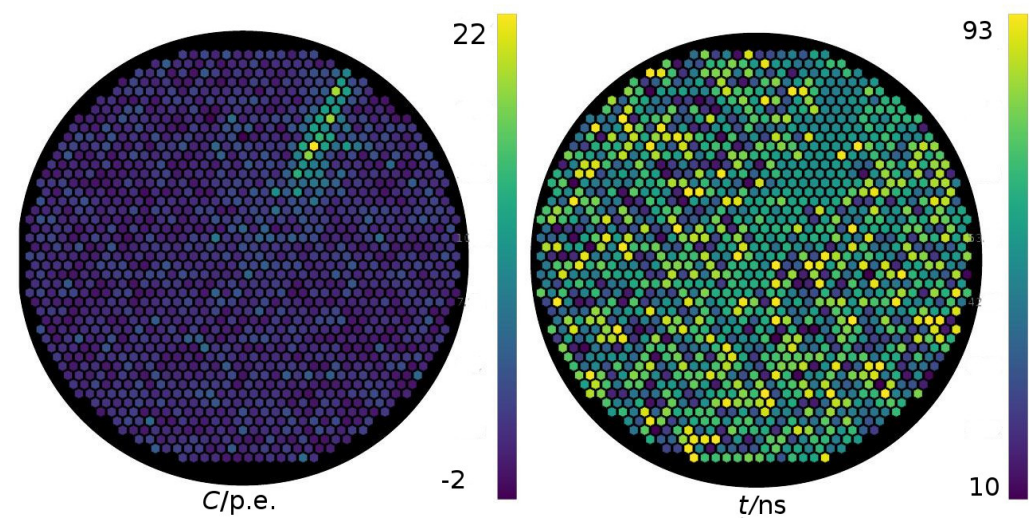

Figure 3: The classic and widely used IACT event representation of photonequivalents of main pulses $C$ (right), and arrival times of main pulses $t$ (left)

\section{Method}

Our SiPM single photon extractor is based on two assumptions. We assume first, that the amplitude of a pixel time line is only the sum of individual avalanche photo diode discharge pulses, and second, that all avalanche photo diode discharge pulses are the same for all pixels, and epochs. We take the recorded time line of a pixel and correlate it with the a rising edge template of a single photon pulse, see Figure 4. On the resulting smooth response, we identify the maximum correlation and define the associated time $t_{1}$ to be the arrival time of a single photon. We append this arrival time $t_{1}$ to the list of arrival times for this pixel and subtract a full single pulse template from the input time line just at time $t_{1}$, see Figure 5. The remaining time line is further processed until all pulses were subtracted. In this loop of extractions, all single photon arrival times are identified on a pixel time line. We are optimistic that our single pulse extractor can be implemented close to the telescope hardware, however this implementation here is in post and software.

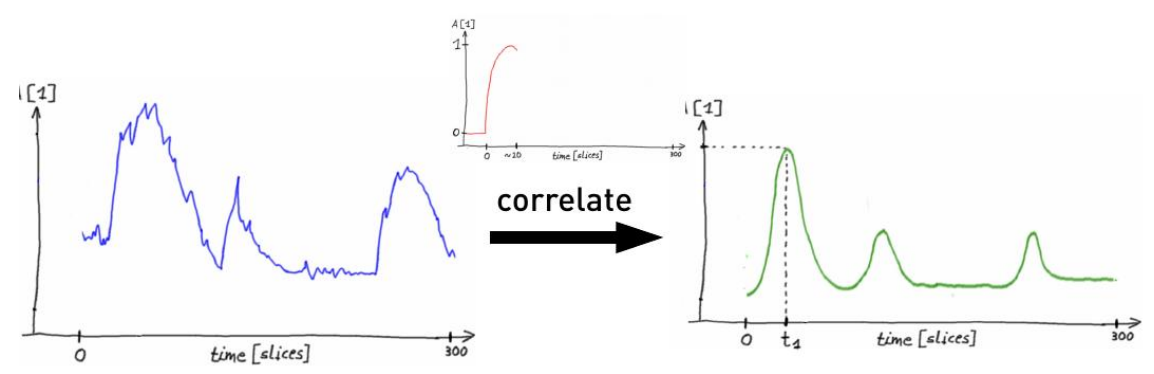

Figure 4: The pixel time line is correlated with the SiPM single pulse template. For the correlation we use the rising edge and the top of the pulse but not its long tail. 


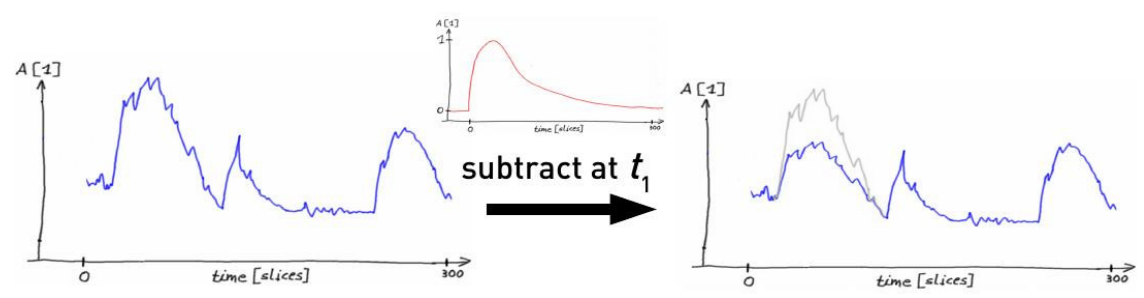

Figure 5: The pixel time line is subtracted the full SiPM single pulse template at the positon $t_{1}$ where the correlation found a photon. Bright grey time line is before subtraction.

We run our extractor on simulated time lines with known arrival times. Figure 7 shows the remaining pixel time line after a single photon has been found at time: 31.5, 92, 17, 32.5, 92, 19.5, and $63.5 \mathrm{~ns}$. In the end, the time line is flat and all pulses were subtracted. The electronics white noise and the photon rate of $50 \mathrm{MHz}$ are typical for observations with FACT. We estimate the performance and limitations of our novel extractor using its miss rate and its sensitivity for various coincidence time matching window radii, see Figure 6 . We find $\sim 80$ percent sensitivity (true positive rate) for a 1 ns timing precision on single photons. Find all sources on GitHub $[3,4,5]$.

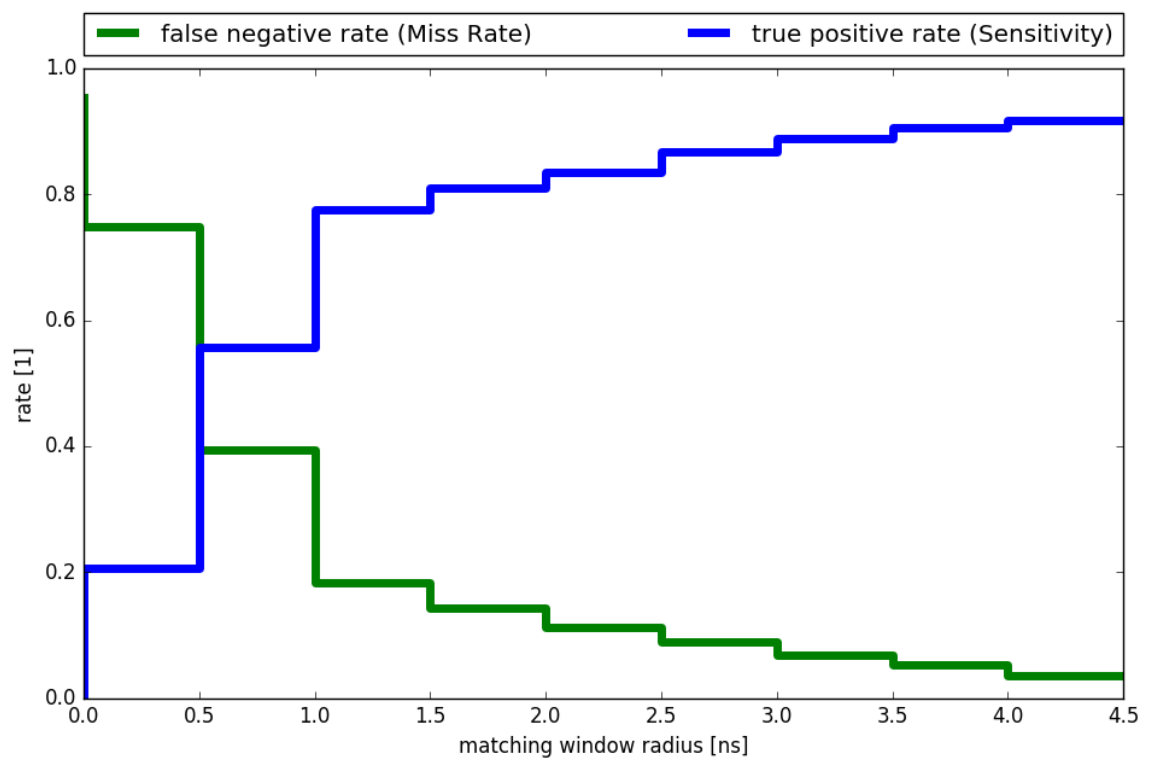

Figure 6: The performance and limitations of our first SiPM single pulse extractor for typical FACT observation conditions based on simulated pixel time lines, see Figure 7. We compare the list of true arrival times with the list of extracted arrival times. For each true arrival time wo look for a match within a matching window radius (here on the $x$-axis) with the nearest extracted arrival time. This way we estimate the number of true positive, false negative and false positive matches while we remove the true positive arrival times from the extracted list in the process. From this we calculate the sensitivity and the miss rate. For each matching window radius we simulated 5000 pixel time lines. A pixel time line is $150 \mathrm{~ns}$ long. 


\section{Results}

Instead of having just one arrival time $t$ and just one photo equivalent $C$ which used to be associated with the main pulse found in each pixel time line, we now have for each pixel a list of arrival times for individual single photons. We call this novel IACT event representation the photon-stream, as it describes the time and structure of photons streaming through the image plane. We plot the single photons, each as a point in the 3D space of the two pixel directions, and the arrival time. Figure 8 shows an actual recorded event by FACT in photon-stream representation. In this 3D space of two directions and one time, we use spatial density clustering (DBSCAN algorithm [6]) to identify air-shower photons in the pool of night sky background photons. Figure 9 shows a second air-shower example event. See also our video animation [7].

\section{Outlook and Conclusion}

We investigate to lower the energy threshold of FACT by explicitly going to single photons and using the density clustering in the photon-stream instead of conventional image cleaning. We explore improving gamma hadron separation by using the novel timing information in the photon-stream. We profit already now from a dramatically reduced file size of the photon-stream which is about $3.7 \mathrm{kByte} / \mathrm{event}$ and thus almost two orders of magnitude smaller than our usual raw data. In the dawn of the Cherenkov Telescope Array (CTA) and its many SiPM based telescopes, we are optimistic that the photonstream is a great opportunity for future airshower reconstruction and raw-event storage.
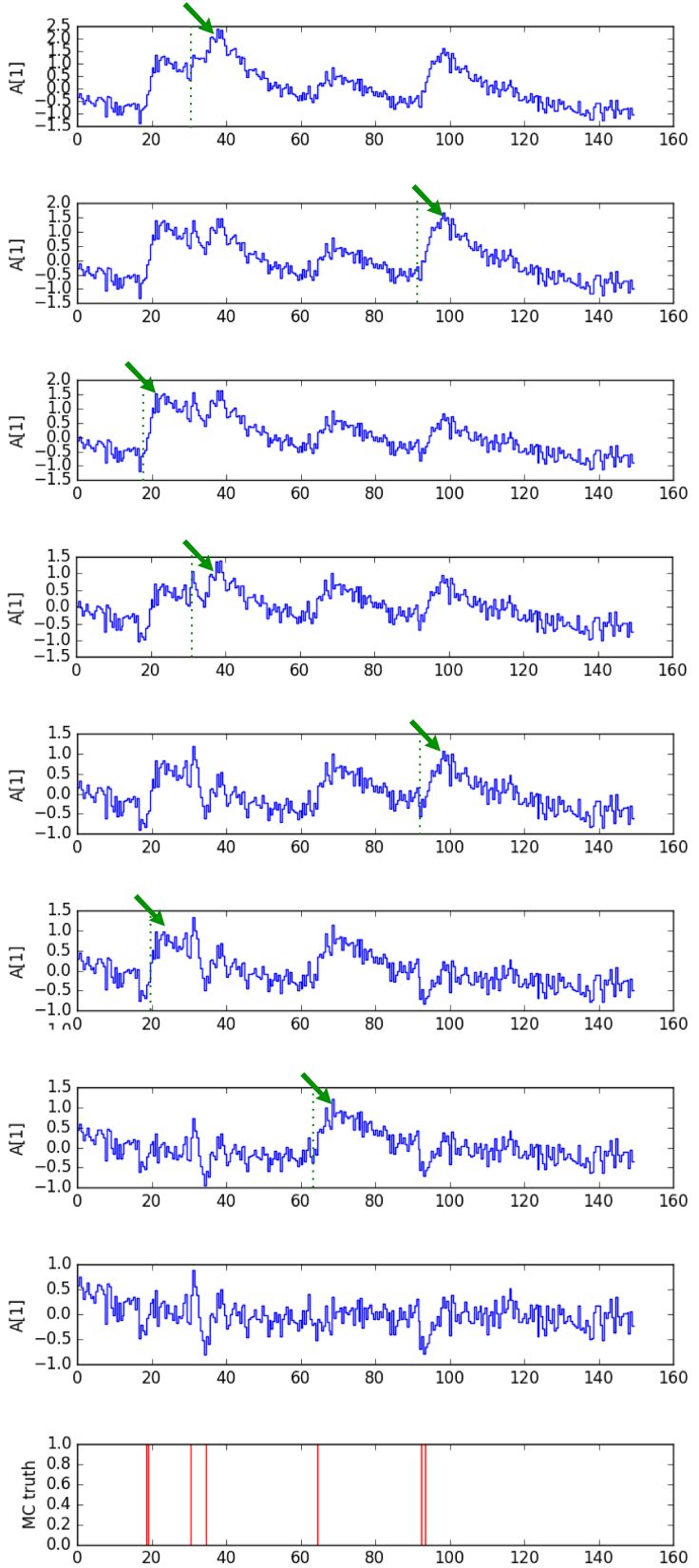

Figure 7: Amplitude time line of a pixel after each single photon extraction iteration. At the bottom in red is the true arrival time of the injected photons. $x$-axis is the time in nano seconds. Green dashed line and arrows indicate subtraction position. Extractions at: 31.5, 92, 17, 32.5, 92, 19.5, and $63.5 \mathrm{~ns}$. 
Night 20151113, Run 109, Event 18
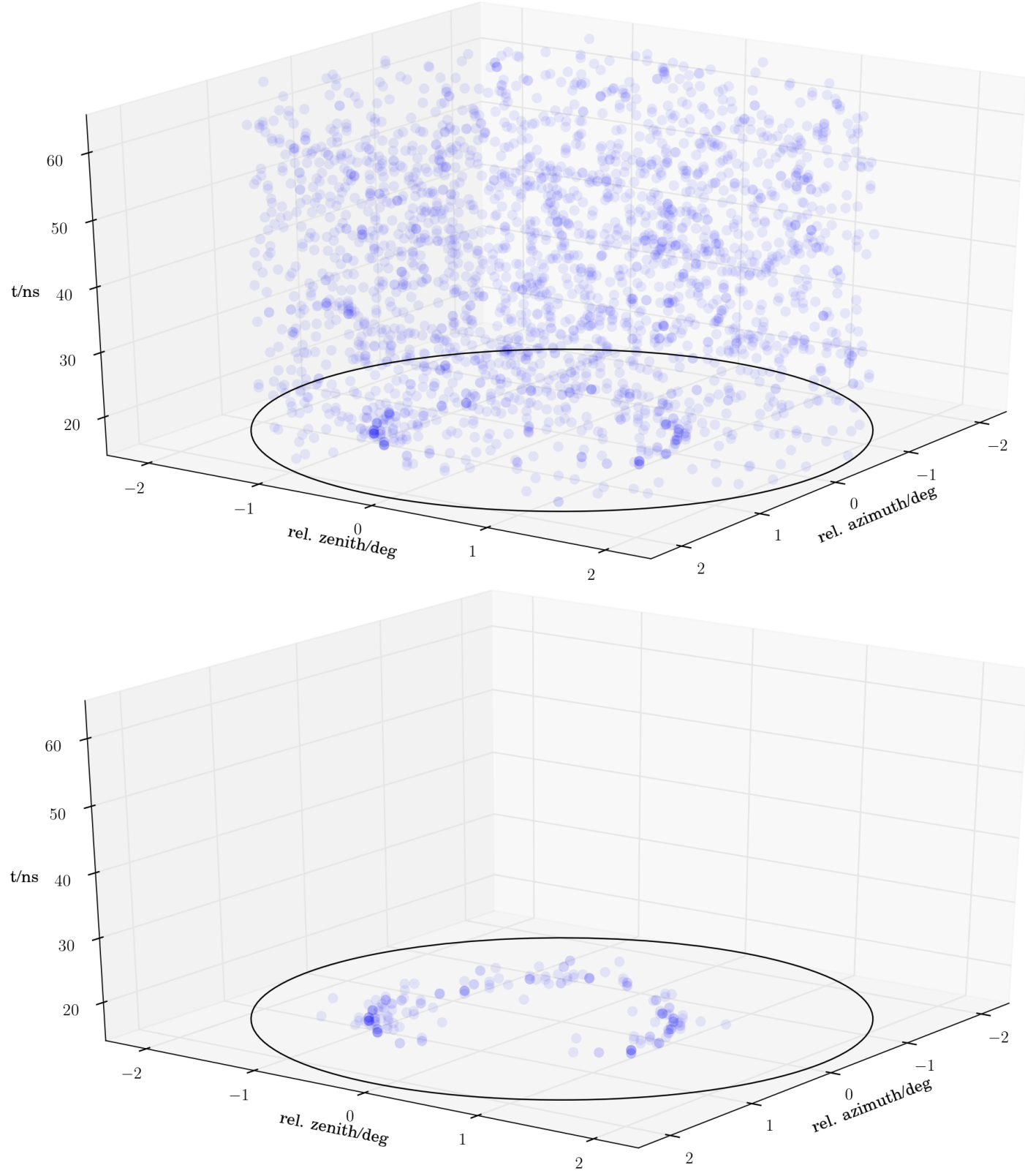

Figure 8: An example event recorded by FACT in photon-stream representation. The upper part shows the full raw photon-stream as it was recorded by FACT and processed by our single photon extractor. The lower part shows the same event, but only the photons which are identified as air-shower Cherenkov photons based density clustering (DBSCAN). Most probably this event was induced by a near by muon which gives the ring structure. The black ring at the bottom of the axes indicates FACT's field of view of $4.5^{\circ}$. A darker blue indicates more photons, the photon markers are semi-transparent. There are 196 photons in 85 pixels in the remaining cluster and 36 pixels which only contain one single photon. 

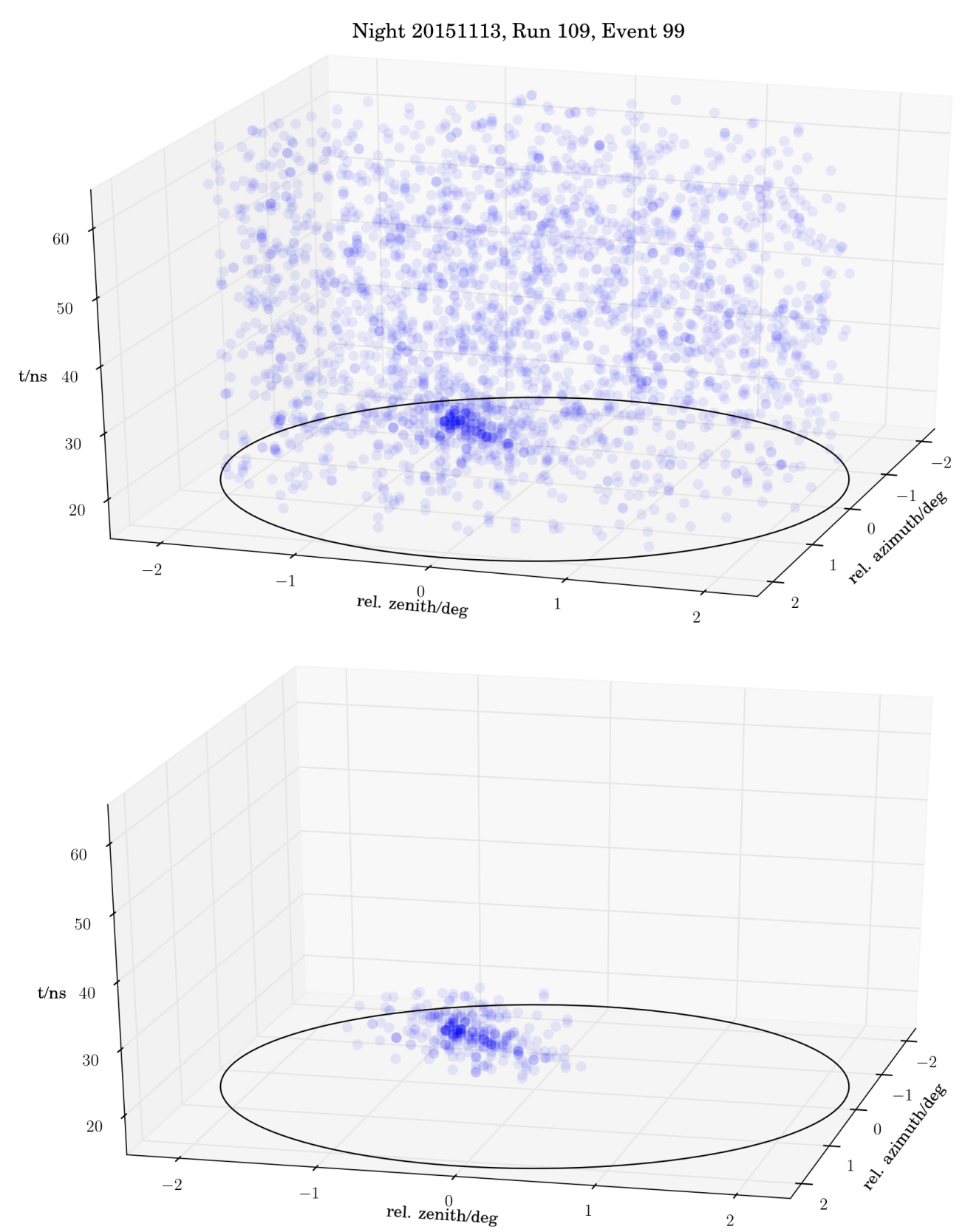

Figure 9: A second example event recorded by FACT in photon-stream representation. Again we identify air-shower Cherenkov photons using density clustering (DBSCAN) in the photon-stream. Most probably an air-shower induced by a proton. There are 347 photons in 123 pixels in the remaining cluster and 54 pixels which only contain one single photon. 
Acknowledgment The important contributions from ETH Zurich grants ETH-10.08-2 and ETH-27.12-1 as well as the funding by the Swiss SNF and the German BMBF (Verbundforschung Astro- und Astroteilchenphysik) and HAP (Helmoltz Alliance for Astroparticle Physics) are gratefully acknowledged. Part of this work is supported by Deutsche Forschungsgemeinschaft (DFG) within the Collaborative Research Center SFB 876 "Providing Information by Resource-Constrained Analysis", project C3. We are thankful for the very valuable contributions from E. Lorenz, D. Renker and G. Viertel during the early phase of the project. We thank the Instituto de Astrofísica de Canarias for allowing us to operate the telescope at the Observatorio del Roque de los Muchachos in La Palma, the Max-Planck-Institut für Physik for providing us with the mount of the former HEGRA CT3 telescope, and the MAGIC collaboration for their support

\section{References}

[1] H. Anderhub et al. "Design and operation of FACT - the first G-APD Cherenkov telescope". In: JINST 8.06 (2013), P06008.

[2] A. Biland et al. "Calibration and performance of the photon sensor response of FACT - the first G-APD Cherenkov telescope". In: JINST 9.10 (2014), P10012.

[3] S. A. Mueller, D. Neise, and J. Buss. Photon-Stream GitHub-Repository. URL: https : //github.com/fact-project/photon_stream.

[4] S. A. Mueller, D. Neise, and M. Noethe. single-pulse-extractor GitHub-Repository. URL: https://github.com/fact-project/single_photon_extractor.

[5] M. L. Ahnen et al. FACT-Tools GitHub-Repository. URL: https : / / github . com/ factproject/fact-tools.

[6] F. Pedregosa et al. "Scikit-learn: Machine Learning in Python". In: Journal of Machine Learning Research 12 (2011), pp. 2825-2830.

[7] FACT collaboration. Photon-Stream animation on YouTube. URL: https : / / www • yout ube . $\mathrm{com} /$ watch? $\mathrm{v}=\mathrm{F} 0 \mathrm{c} 2 \mathrm{tcj} 1 \mathrm{rb} 8$. 